\title{
Range of Motion of the Upper Cervical Spine: Flexion, Extension, Lateral Bending, and Axial Rotation
}

Ana I. Lorente ${ }^{1}$, César Hidalgo García ${ }^{2}$, Jacobo Rodríguez Sanz ${ }^{3}$, Mario Maza Frechín ${ }^{1}$, Albert Pérez Bellmunt ${ }^{3}$

1 . Impact Laboratory

UniversityofZaragoza

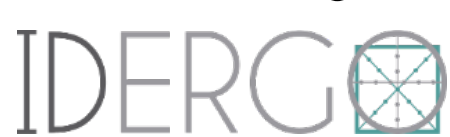

2 || U.I.F.

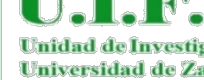

3 UĹ

Clinical evaluation of upper cervical spine instability with manual therapy $\Rightarrow$ Normal range of movement?

In vitro simulation of the clinical test: 10 upper cervical spine specimens

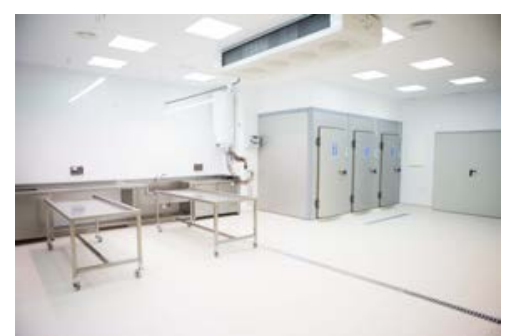

Impact Laboratory (I3A), Alcañiz

\section{Introduction}

- Common symptom in adults: neck pain

- Manual therapy to examine patients: screening cervical spine instability

\section{Objective}

To quantify motion and applied torque

\section{Methodology}

Hidalgo et al., 2020. Musculoskelet. Sci. Pract.

- Motion capture system (Vicon)

- Measure device (Faro)

Coordinates of Vicon markers and anatomical landmarks $\rightarrow$ Coordinate systems to quantify the motion

- Force and torque sensor (AMTI)

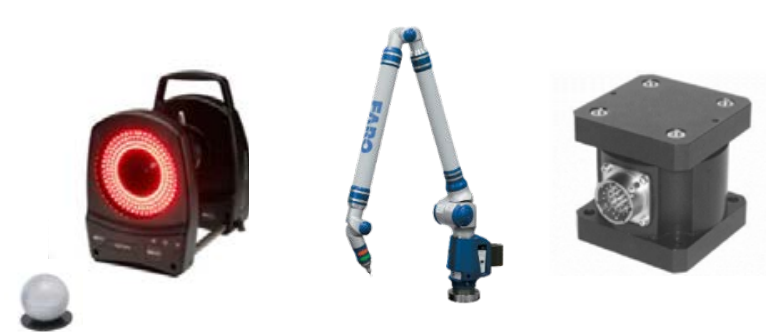

Results

\begin{tabular}{lcc} 
& ROM $\left(^{\circ}\right)$ & Torque $(\mathbf{N m})$ \\
\hline Flexion & $19.8 \pm 5.3$ & $0.7 \pm 0.2$ \\
Extension & $14.4 \pm 7.7$ & $0.9 \pm 0.3$ \\
\hline LB - R & $4.7 \pm 2.3$ & $0.8 \pm 0.2$ \\
LB - L & $5.6 \pm 3.2$ & $1.0 \pm 0.3$ \\
\hline Rot - R & $33.9 \pm 6.6$ & $0.6 \pm 0.1$ \\
Rot - L & $28.0 \pm 6.9$ & $0.6 \pm 0.2$
\end{tabular}

ROM: range of motion; LB: lateral bending; Rot: axial rotation;

\section{Conclusions}

- Valuable results from a clinical point of view: first in vitro tests with C2 fixed (as in manual therapy) 